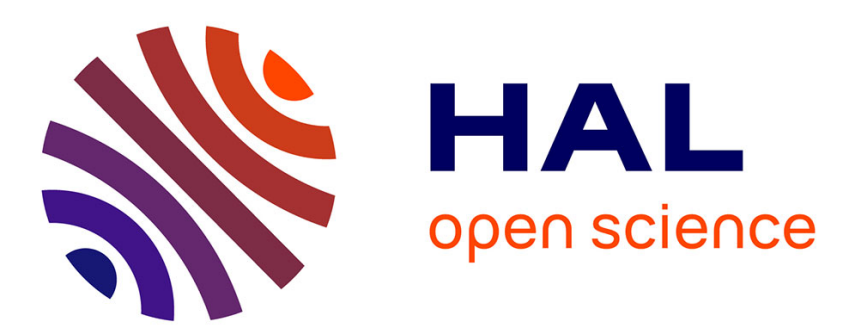

\title{
La gestion des populations. Comment exploiter la variabilité génétique entre races : du croisement simple à la souche synthétique \\ Jean Pierre Bidanel
}

\section{- To cite this version:}

Jean Pierre Bidanel. La gestion des populations. Comment exploiter la variabilité génétique entre races : du croisement simple à la souche synthétique. Productions Animales, 1992, 1992, pp.249-254. 10.20870/productions-animales.1992.5.HS.4299 . hal-02712138

\section{HAL Id: hal-02712138 \\ https: / hal.inrae.fr/hal-02712138}

Submitted on 1 Jun 2020

HAL is a multi-disciplinary open access archive for the deposit and dissemination of scientific research documents, whether they are published or not. The documents may come from teaching and research institutions in France or abroad, or from public or private research centers.
L'archive ouverte pluridisciplinaire HAL, est destinée au dépôt et à la diffusion de documents scientifiques de niveau recherche, publiés ou non, émanant des établissements d'enseignement et de recherche français ou étrangers, des laboratoires publics ou privés. 


\section{J.-P. BIDANEL}

INRA Station de Génétique quantitative et appliquée 78352 Jouy-en-Josas Cedex
La gestion des populations

\section{Comment exploiter la variabilité génétique entre races : du croisement simple à la souche synthétique}

Résumé. Cet article fait le point sur les modalités d'exploitation par le croisement de la variabilité génétique entre races. Le croisement ou accouplement d'animaux issus de populations différentes permet de tirer parti des différences additives entre races, de bénéficier des effets d'hétérosis et de complémentarité et peut dans certains cas conduire à un accroissement de la variabilité génétique. Le croisement recouvre en fait un grand nombre de modalités appelées systèmes ou plans de croisement. Ceux-ci different entre eux par le nombre de populations impliquées et le mode de renouvellement des reproducteurs, mais également par l'utilisation des différences entre races, des effets d'hétérosis et de complémentarité. Le choix d'un plan de croisement dépend des caractéristiques biologiques de l'espèce, des conditions socio-économiques de production et bien entendu de son efficacité économique. Celle-ci est fonction des effectifs et des performances moyennes des différents types génétiques produits. Ces performances moyennes peuvent être prédites à un instant $t$ à partir des paramètres du croisement (effets additifs et d'hétérosis directs et maternels). La valeur additive d'un type génétique se calcule alsément comme la somme des effets additifs de chaque race pondérés par leur proportion relative dans le génotype considéré. La prédiction des effets d'hétérosis peut s'avérer plus complexe, car elle dépend de la nature des effets d'hétérosis (dominance ou épistasie).Par contre, pour pouvoir prédire la performance moyenne d'un type génétique au cours du temps, il est nécessaire de prendre en compte les effets conjoints de la sélection et du croisement.

La variabilité génétique entre races représente une part très importante de la variabilité génétique totale dans la plupart des espèces d'animaux domestiques. De façon similaire à la situation intra-race, cette variabilité peut être d' origine additive (différences génétiques entre races) ou non additive (effets d'hétérosis générés par le croisement). Elle peut être due aux effets des gènes de l'individu sur lequel est mesurée la performance. On parle alors d'effet d'effet additif direct et d'hétérosis direct. Elle peut également être due aux effets des gènes de la mère de cet individu. On parle alors d'effet additif maternel et d'hétérosis maternel.

Le croisement, ou accouplement entre des individus appartenant à des populations (races ou lignées) génétiquement distinctes, constitue l'outil de base permettant d' exploiter la variabilité génétique entre races. Ce terme générique recouvre en fait un grand nombre de variantes appelées systèmes de croisement ou plans de croisement qui different entre elles par le nombre de populations impliquées et le mode de renouvellement des reproducteurs. L'exemple le plus immédiat est celui du croisement simple, où des individus de deux populations sont accouplés pour produire des animaux F1 destinés à l'abattage. Mais des situations plus complexes peuvent exister. Chez le porc, un système de croisement assez répandu utilise des verrats et des truies qui sont eux-mêmes croisés pour la production des animaux destinés à l'abat- tage. Ce plan de croisement, qui implique quatre populations grand-parentales, est appelé croisement à quatre voies.Ce ne sont là que deux exemples parmi d'autres. Cet article fait le point sur les différentes modalités d'exploitation de la variabilité génétique entre races. Après une présentation des objectifs du croisement et des principaux systèmes de croisement, les éléments permettant de raisonner le choix d'un système de croisement sont exposés.

\section{1 / Les objectifs du croisement}

On peut distinguer quatre objectifs au croisement, qui ne sont pas recherchés de façon simultanée dans tous les plans de croisement.

\section{1 / Exploitation du phénomène d'hétérosis}

Le gain dû à l'hétérosis varie selon les populations parentales. Il est d'autant plus important que la distance entre les populations est élevée. Ainsi, l'hétérosis du croisement entre les deux races porcines Meishan (d'origine chinoise) et Large White, génétiquement très éloignées, est, selon le caractère, de deux à quatre fois plus élevé qu'entre les deux races Large White et Landrace français, génétiquement assez proches. L'hétérosis varie également selon le 
caractère. Il est en général inexistant pour les caractères de composition corporelle, mais peut dépasser $10 \%$ de la moyenne des races parentales pour les caractères de reproduction. Il dépend aussi du système de croisement. Par exemple, le croisement simple décrit ci-dessus ne permet d'exploiter que les effets d'hétérosis direct sur la croissance et l'efficacité alimentaire. Le croisement à quatre voies permet quant à lui d'exploiter ces mêmes effets d'hétérosis direct, mais également les effets d'hétérosis maternel sur les caractères de reproduction des femelles. Nous y reviendrons ultérieurement.

\section{2 / Exploitation du phénomène de complémentarité}

Le croisement permet également de réunir, chez un même animal, des aptitudes complémentaires présentes soit dans une race, soit dans l'autre, mais difficiles à réunir par sélection dans une seule race; c'est la complémentarité. Il est par exemple en général intéressant, pour la production de viande, d'accoupler une race paternelle à fort développement musculaire à une race maternelle possédant de bonnes performances d'élevage et de reproduction. Une situation particulière, mais proche de la précédente quant au principe, concerne le cas d'un gène majeur où l'hétérozygote est le génotype économiquement le plus performant. On cherchera alors à fixer un allèle différent dans chaque population parentale de façon à obtenir des descendants croisés hétérozygotes.

\section{3 / Utilisation des différences additives entre races}

Le croisement peut aider à tirer parti de la supériorité d'une race sur une autre race pour un caractère ou un ensemble de caractères. Supposons qu'un éleveur de vaches laitières de race Française Frisonne souhaite les remplacer par des animaux de race Holstein, plus productives. Il peut le faire en vendant son cheptel et en achetant des vaches Holstein ou en pratiquant des transplantations d'embryons Holstein. Mais il peut également, de façon plus économique, croiser par insémination artificielle toutes ses vaches avec des taureaux Holstein. $\mathrm{Au}$ bout de sept générations, le génotype de ses femelles sera Holstein à plus de $99 \%$. Ce type de croisement, appelé croisements en retour successifs ou croisement d'absorption peut également être utilisé pour introduire un gène majeur présent dans une population dans une autre population qui en est dépourvue. Ainsi, le gène Booroola, associé à une forte prolificité chez le mouton, est présent dans une population économiquement peu intéressante du fait de son faible potentiel de croissance musculaire. On montre qu'il est nettement plus efficace d'introduire le gène Booroola dans une race à fort potentiel de croissance musculaire par croisements en retour successifs que de chercher à sélectionner la race Booroola.

\section{4 / Accroissement de la variabilité génétique}

La création d'une nouvelle race ou population à partir de races possédant des qualités complémentaires peut dans certains cas être une alternative intéressante au maintien et au croisement des populations parentales. Dans le cas le plus simple, on par- tira de deux races que l'on croisera. Les animaux F1 seront accouplés entre eux pour produire les F2, les F2 pour produire les F3,etc. Une telle population est dite synthétique ou composite. Elle conduit à une situation plus simple à gérer sur le plan génétique. Elle peut dans certains cas permettre une augmentation de la variabilité génétique. Cette augmentation est cependant loin d'être systématique et ne peut en général pas être prédite, de sorte qu'elle ne peut à elle seule pas justifier la création d'une population synthétique. Par contre, la réunion, chez un même animal, d'aptitudes complémentaires permet souvent d'élargir l'éventail des combinaisons de caractères et d'envisager une sélection plus efficace des combinaisons les plus favorables.

\section{2 / Les différents systèmes de croisement}

On distingue en général deux grandes catégories de systèmes de croisement, les croisements discontinus ou statiques, qui visent à obtenir une génération d'animaux destinés en totalité à l'abattage et pour lesquels il faut obtenir et renouveler les reproducteurs de race pure, et les croisements continus, qui produisent leurs propres reproducteurs. Des systèmes "mixtes" combinant les deux approches, peuvent toutefois exister. On considérera, à titre d'exemple, les quatre races porcines Hampshire $(\mathrm{H})$, Landrace $(\mathrm{L})$, Piétrain $(\mathrm{P})$ et Large White ou Yorkshire (Y).

\section{1 / Les plans de croisements discontinus}

Ils differrent selon le nombre de races concernées et de générations de croisement nécessaires pour obtenir les produits terminaux. Dans le cas évoqué ci-dessus du croisement simple (encore appelé croisement à 2 voies ou croisement "industriel"), les produits terminaux sont obtenus en une seule génération (figure 1).

Figure 1. Principaux systèmes de croisement discontinus.

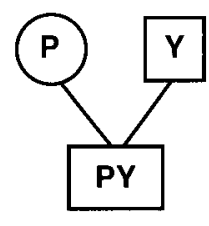

à 2 voies

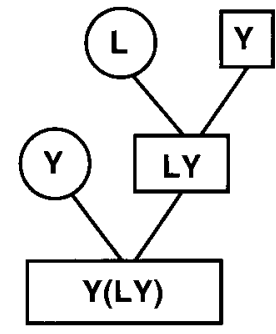

en retour

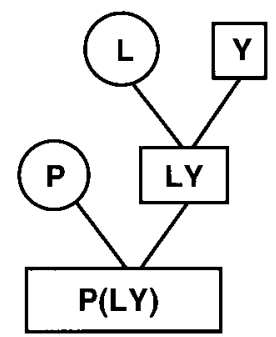

à 3 voies

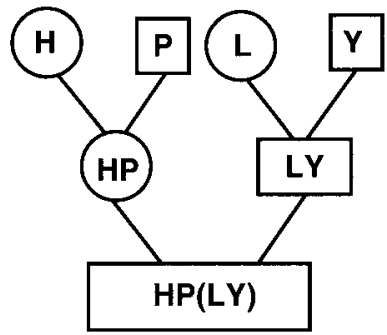

à 4 voies 
On parle de croisement à double étage lorsque les produits terminaux sont obtenus après deux générations de croisement. Des femelles et/ou des mâles issus d'une première génération de croisement sont utilisés comme reproducteurs pour produire les animaux destinés à l'abattage. On distingue classiquement trois types de plans de croisement à double étage selon les nombre de races concernées (figure 1). Le croisement à quatre voies, déjà évoqué, dans lequel les génotypes parentaux sont tous deux croisés, implique en général quatre races. Une variante à trois races, où une des races est commune aux deux génotypes parentaux est parfois utilisée. C'est le cas par exemple chez le porc avec le croisement $(\mathrm{PxY}) \mathrm{x}(\mathrm{LxY})$. Dans le croisement à trois voies, l'un des parents est croisé (en général la femelle) et accouplé à un animal d'une troisième race. Enfin, dans le croisement en retour, le génotype parental de race pure est également utilisé pour produire le génotype parental croisé.

\section{2 / Les plans de croisement continus}

Dans les plans de croisement continus, une partie des animaux croisés est gardée pour produire la génération suivante. Il n'y a donc plus à proprement parler de génération terminale. Certains plans de croisement continus, à savoir le croisement d'absorption et la constitution d'une lignée composite, ont déjà été décrits de façon détaillée. Nous n'y reviendrons pas. Un autre type, le croisement d'amélioration, est proche du croisement d'absorption. Il consiste en une utilisation, non plus systématique mais limitée, d'une race, en vue d'améliorer de façon modérée les performances d'une autre race moins productive. Mais la forme la plus répandue de croisement continu reste le croisement rotatif. Son principe consiste à remplacer à chaque génération les reproducteurs de l'un des deux sexes (généralement les femelles) par des animaux issus du croisement et à les accoupler à des animaux de race pure, celle-ci changeant à chaque génération (figure 2 ). Un croisement rotatif à deux races est également appelé croisement alternatif.

\section{3 / Les plans de croisement "mixtes"}

Il s'agit en général de plans de croisement discontinus, car les produits de la dernière génération de croisement sont tous abattus, mais avec l'un au moins des parents, en général la mère, issu d'un plan de croisement continu (alternatif ou rotatif). Il est alors possible de tirer parti des effets de complémentarité et d'hétérosis tout en assurant un autorenouvellement des femelles.

\section{3 / Comparaison des systèmes de croisement}

Les systèmes de croisement diffêrent quant à l'utilisation de l'hétérosis, de la complémentarité et des différences additives entre races. Aucun système n'est systématiquement supérieur aux autres. En général, les croisements discontinus utilisent mieux les phénomènes de complémentarité (par l'utilisation de types génétiques paternels et maternels spécialisés) et d'hétérosis. En revanche, ils sont souvent plus compliqués à gérer, notamment sur les plans génétique et sanitaire, et de ce fait plus coûteux. De plus, le problème du renouvellement des femelles dans les noyaux de race pure limite fortement leur développement dans les espèces à faible productivité numérique (bovins et dans une moindre mesure ovins). En effet, seul le nombre de femelles en excès par rapport à celui nécessaire pour renouveler les noyaux de race pure est susceptible d'être de génotype croisé. Ainsi, dans l'espèce bovine, la proportion de femelles disponibles pour produire des descendants croisés ne peut, en l'absence de contrôle du sexe, guère excéder $50 \%$ dans le meilleur des cas. Les croisements continus, dans lesquels le renouvellement des femelles est beaucoup plus aisé, possèdent de ce fait de nombreux atouts dans ces espèces. A l'inverse, le renouvellement des femelles ne pose pas problème dans les espèces à forte productivité numérique et les plans de croisement discontinus à double étage, qui permettent d'utiliser au mieux les effets d'hétérosis, sont en règle générale utilisés.

Malgré ces quelques principes de base, le nombre de plans de croisement envisageable dans une espèce donnée reste en général très élevé. En pratique, le choix d'un système de croisement pourra dépendre des conditions socio-économiques et d'organisation de la production, des contraintes de gestion de ce système et bien entendu de son efficacité économique. Celle-ci dépend étroitement des performances des différents types génétiques produits dans le plan de croisement considéré.

Figure 2. Principaux systèmes de croisement continus.

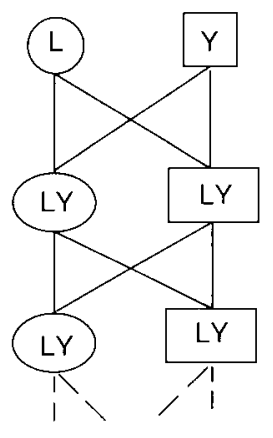

lignée synthétique

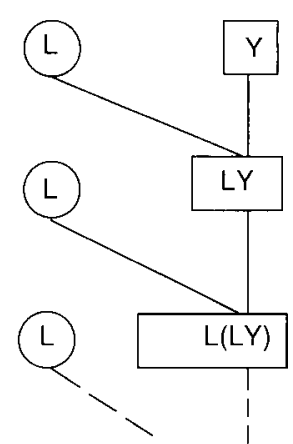

d'absorption
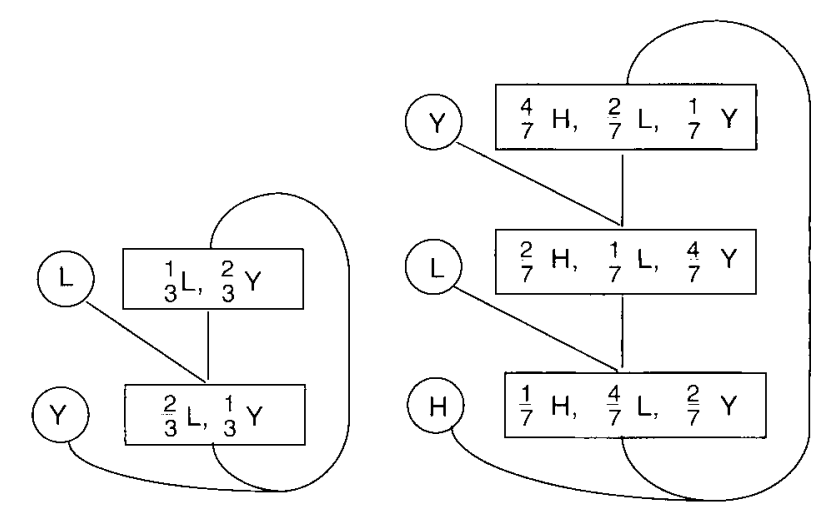

alternatif

rotatif à 3 races 


\section{1 / Prédiction des performances en croisement}

Les performances en croisement sont le plus souvent caractérisées par leurs seules valeurs moyennes. La variabilité de ces performances peut également être un paramètre important, mais, sauf cas particulier, la variabilité phénotypique des produits croisés est similaire à ce qu'elle est intra-race, de sorte que l'on s'y intéresse en général assez peu. Les performances moyennes d'un type génétique peuvent être prédites à partir des paramètres du croisement comme la somme des effets additifs et non additifs entre races. La valeur additive d'un type génétique se calcule très simplement comme la somme des valeurs de chaque race pondérées par leur proportion respective dans le génotype considéré. Ainsi, si l'on reprend l'exemple du croisement à trois voies entre un verrat $P$ et une truie $L x Y$, la valeur additive des animaux $\operatorname{Px}(\mathrm{Lx} Y)$ s'écrira:

$$
G=1 / 2 g_{P}^{0}+1 / 4\left(g_{L}^{0}+g_{Y}^{0}\right)+1 / 2\left(g_{L}^{m}+g_{Y}^{m}\right)
$$

Cette expression se généralise aisément à un plan de croisement quelconque :

$$
G=\Sigma_{i} p_{i}^{\circ} g_{i}^{\circ}+\Sigma_{i} p_{i}^{m} g_{i}^{m}
$$

où $P_{i}^{o}$ et $P_{i}^{m}$ sont les proportions de la race i dans le génotype croisé et chez sa mère, $g_{i}^{o}$ et $g_{i}^{m}$ les valeurs additives directe et maternelle de la race $i$.

La valeur de l'hétérosis est plus difficile à calculer, notamment dans les systèmes de croisement complexes. Elle dépend du type d'interaction entre gènes (dominance ou épistasie) à l'origine du phénomène d'hétérosis. Chez un animal F1, l'hétérosis est dans tous les cas maximal car toutes les paires de chromosomes sont constituées de chromosomes d'origine différente. Il y a donc possibilité d'hétérozygotie et par conséquent de dominance pour l' ensemble des locus du génome. De la même façon, dans les autres croisements, l'hétérosis dépendra de la proportion de locus pour lesquels il y a possibilité d'hétérozygotie. Chez un animal F2 de constitution (PxY)x(PxY), il y aura possibilité d'hétérozygotie aux locus possédant un allèle d'origine $\mathrm{P}$ et l'autre d'origine $\mathrm{Y}$. Cette situation se présentera si le père transmet un allèle d'origine $\mathrm{P}$ et la mère un allèle d'origine $\mathrm{Y}$ ou le père un allèle d'origine $Y$ et la mère un allèle d'origine $P$, soit avec une probabilité 1/2. L'hétérosis direct en F2 est donc la moitié de sa valeur en F1. L'hétérosis maternel est quant à lui maximal car la mère est F1. Dans le cas d'un croisement à trois voies $\mathrm{Px}(\mathrm{LxY})$, l'hétérosis maternel est maximal pour la même raison qu'en F2. Chez les individus de génotype $\operatorname{Px}(\mathrm{LxY})$, il y a possibilité d'hétérozygotie pour l'ensemble des locus, mais il s'agira dans 50\% des cas d'interactions entre allèles d'origine $\mathrm{P}$ et $\mathrm{L}$ et dans $50 \%$ d'interactions entre allèles d'origine $P$ et $Y$. Les interactions et donc l'hétérosis ne sont pas nécessairement les mêmes dans les deux cas et la valeur globale de l'hétérosis s'écrira :

$H^{0}=0,5 h_{P L}^{0}+0,5 h_{P Y}^{\circ}$

où $h_{\mathrm{PL}}^{\circ}$ et $h_{\mathrm{PY}}^{\circ}$ sont les valeurs de l'hétérosis dans les croisements PxL et PxY respectivement.
Dans le cas d'un croisement quelconque entre un type génétique paternel constitué de $p$ races de proportions respectives $p_{i}$ et un type génétique maternel constitué de $q$ races de proportions respectives $q_{j}$, l'hétérosis global s'écrira :

$\mathrm{H}=\Sigma_{i} \Sigma_{\mathrm{i}} \mathrm{p}_{\mathrm{i}} \mathrm{q}_{\mathrm{i}} \mathrm{h}_{\mathrm{ij}}$

où $h_{i j}$ est l'héterosis du croisement entre les deux races i et j. Les effets d'hétérosis pour les principaux plans de croisement continus et discontinus figurent dans le tableau 1.

Tableau 1. Effets d'hétérosis dans les principaux plans

\begin{tabular}{|c|c|c|c|}
\hline Plan de croisement & (1) & Hétérosis direct & $\begin{array}{l}\text { Hétérosis } \\
\text { maternel }\end{array}$ \\
\hline Simple & $\mathrm{AxB}$ & $h_{\mathrm{AB}}^{\circ}$ & - \\
\hline A trois voies & $\mathrm{A} \times \mathrm{BC}$ & $1 / 2\left(h_{A B}^{\circ}+h_{A C}^{\circ}\right)$ & $h_{B C}^{m}$ \\
\hline En retour & $\mathrm{A} \times \mathrm{AB}$ & $1 / 2 h_{A B}^{\circ}$ & $h_{A B}^{m}$ \\
\hline A quatre voies & $\mathrm{CD} \times \mathrm{AB}$ & $1 / 4\left(h_{A C}^{o}+h_{A D}^{o}+h_{B C}^{o}+h_{B D}^{o}\right)$ & $h_{\mathrm{AB}}^{m}$ \\
\hline A quatre voies & $A C \times A B$ & $1 / 4\left(h_{\mathrm{AB}}^{\circ}+h_{\mathrm{AC}}^{\circ}+h_{\mathrm{BC}}^{\circ}\right)$ & $h_{A B}^{m}$ \\
\hline Alternatif & $\mathrm{Al}(\mathrm{A}, \mathrm{B})$ & $2 / 3 h_{\mathrm{AB}}^{\circ}$ & $2 / 3 h_{A B}^{m}$ \\
\hline Rotatif (2) & $\mathrm{R}(\mathrm{A}, \mathrm{B}, \mathrm{C})$ & $4 / 7 h_{X Y+2 / 7}^{0} h_{X Z}^{0}$ & $4 / 7 \mathrm{~h}_{Y Z^{+2}}^{\mathrm{m}}{ }^{2 / 7 \mathrm{~h}_{X Y}^{m}}$ \\
\hline $\begin{array}{l}\text { Lignée synthétique } \\
\text { à } 2 \text { races }\end{array}$ & $S(A, B)$ & $1 / 2 h_{A B}^{\circ}$ & $1 / 2 h_{A B}^{m}$ \\
\hline
\end{tabular}
de croisement

(1) génotype du père $x$ génotype de la mère

(2) $X, Y$ et $Z$ sont successivement $A, B$ et $C$ au cours des générations

Lorsque l'hétérosis observé en F1 n'est pas uniquement du à des effets de dominance, mais comporte également des interactions entre locus (épistasie), l'hétérosis dans les plans de croisement complexes ne peut plus être prédit à partir de la seule valeur de l'hétérosis en F1. Il est alors nécessaire de connaître les composantes de dominance et d'épistasie de l'hétérosis. La décomposition de l'hétérosis dépendra du type d'interaction épistatique (nombre de locus et type d'effets impliqués) et s'avère vite extrêmement complexe de telle sorte que l'on est en pratique amené à se limiter aux interactions épistatiques les plus simples entre locus pris deux à deux.Même ainsi, une estimation suffisamment précise des effets épistatiques pose souvent problème. Néanmoins, si l'on suppose connues les différentes composantes de l'hétérosis, la prédiction des performances moyennes de types génétiques complexes ne pose pas de problème particulier. A titre d'exemple, les équations de prédiction des performances moyennes de quelques types génétiques en présence d'interactions épistatiques de type additif $\mathrm{x}$ additif sont présentées dans le tableau 2 .

\section{2 / Critères de comparaison}

Une fois connues les performances moyennes des différents types génétiques d'un plan de croisement, il reste à définir un critère global d'appréciation de son efficacité économique. Il s'agira d'une fonction plus ou moins complexe des performances moyennes de tout ou partie des types génétiques produits dans le plan de croisement. Il peut s'agir d'une fonction extrêmement simple, comme le bénéfice (recettes - 
Tableau 2. Prédiction des effets non additifs en présence d'interactions épistatiques: quelques exemples simples (d'après Koch et al 1985).

\begin{tabular}{|c|c|c|}
\hline Type génétique & Effets directs $(1)$ & Effets maternels $(1)$ \\
\hline $\begin{array}{l}\text { Croisement } \\
\text { simple } A \times B\end{array}$ & $d^{0}+1 / 2 a a^{O}$ & $a a^{m}$ \\
\hline $\begin{array}{l}\text { Croisement en } \\
\text { retour } \mathrm{AxAB}\end{array}$ & $1 / 2 d^{0}+5 / 8 a a^{0}$ & $d^{m}+1 / 2 a a^{m}$ \\
\hline $\begin{array}{l}\text { Lignée synthétique } \\
\text { à } 2 \text { races } S(A, B)\end{array}$ & $1 / 2 d^{O}+1 / 2 a a^{O}$ & $1 / 2 d^{m}+1 / 2 a a^{m}$ \\
\hline
\end{tabular}

(1) $d^{0}, d^{m}, a a^{0}, a a^{m}$ : effets de dominance et d'épistasie directs et maternels, respectivement

coûts) par animal au niveau de la génération "terminale" du croisement. On suppose alors implicitement que les recettes et coûts non directement imputables aux produits terminaux sont similaires dans les différents plans de croisement. Lorsque cette hypothèse n'est pas vérifiée, un calcul des recettes et des coûts pour chacun des "maillons" du système est nécessaire et le critère de comparaison est alors la somme, pondérée par les effectifs, des bénéfices par animal pour chacun des maillons. Enfin, les plans de croisement peuvent également différer quant à l'efficacité de la sélection pratiquée dans les noyaux de race pure. Il est alors nécessaïre de connaître et de modéliser l'évolution génétique des noyaux de race pure et ses effets sur les performances moyennes des génotypes croisés (quels sont les paramètres du croisement affectés ? Comment sont-ils affectés?). En pratique, on est amené à scinder chacun des maillons du système en cohortes d'animaux homogènes sur le plan génétique (ayant les mêmes performances moyennes). L'évolution génétique des performances de chaque cohorte est prédite à partir des paramètres du croisement et d'équations caractérisant les flux d'animaux et de gènes entre cohortes. Le bénéfice à chaque instant t est comme précédemment calculé comme la somme pondérée par les effectifs des coûts et des recettes dans chacune des cohortes. Le critère de comparaison des plans de croisement est la somme des bénéfices à chaque instant t successifs, généralement pondérés par un coefficient d'actualisation permettant de prendre en compte les délais d'obtention du progrès génétique.

\section{3 / Choix des races}

A priori, le choix des races est fonction de la valeur économique relative des plans de croisement. En pratique, il est cependant en général plus judicieux d'éviter une évaluation de l'ensemble des plans de croisement envisageables et de limiter cette évaluation aux seules races susceptibles de présenter un intérêt en croisement.

Un premier choix sur la base des performances en race pure peut être envisagé. Ce type de choix peut présenter un intérêt lorsque les effets d'hétérosis sont faibles. Il peut par ailleurs permettre de distinguer les races paternelles et maternelles spécialisées.
Par contre, lorsque les effets d'hétérosis sont importants et susceptibles de varier d'un croisement à l'autre, la comparaison des performances en race pure est souvent insuffisante. Il peut alors être intéressant de connaître la valeur moyenne en croisement des différentes races. La valeur moyenne en croisement d'une race $\mathrm{A}$ dépend à la fois de ses effets additifs et de son hétérosis moyen (valeur moyenne de l'hétérosis de tous les croisements impliquant la race $A$ ). Cette valeur moyenne en croisement est appelée aptitude générale à la combinaison ou aptitude générale au croisement de la race A. L'écart entre la valeur de l'hétérosis du croisement entre les 2 races $A$ et $B$ et la moyenne de leur aptitude générale au croisement est appelée aptitude spécifique à la combinaison ou aptitude spécifique au croisement.

Lorsque, pour un caractère donné, les aptitudes spécifiques au croisement sont faibles, il est possible de choisir les races sur la base de leur aptitude générale au croisement. Par contre, lorsque les aptitudes spécifiques au croisement sont importantes, le choix est plus délicat, car il s'agit de sélectionner non plus des races, mais des combinaisons de races. Il est alors nécessaire de connaître, ou de savoir prédire, les valeurs d'hétérosis pour chaque croisement. Une telle prédiction est théoriquement possible. En effet, l'hétérosis étant fonction de la diversité génétique entre races, il suffirait de savoir mesurer cette diversité, à l'aide par exemple de marqueurs génétiques, pour être en mesure de prédire l'hétérosis. Malheureusement, les résultats obtenus jusqu'à présent à partir de marqueurs classiques se sont avérés décevants, mais avec il est vrai peu de marqueurs mal répartis sur le génome. On peut penser que l'utilisation de marqueurs moléculaires, disponibles en plus grand nombre et avec une meilleure répartition sur le génome, permettrait d'aboutir à des résultats plus probants.

\section{4 / Croisement et sélection}

La sélection et le croisement sont deux méthodes complémentaires d'amélioration génétique. Cependant, comme nous l'avons évoqué au paragraphe 3.2 , la sélection pratiquée dans les noyaux de race pure affecte de diverses façons les performances en croisement. Inversement, le fait d'utiliser des races en croisement n'est pas sans effet sur l'orientation de la sélection dans ces races.

\section{1 / Effets de la sélection sur la variabilité entre races}

La sélection est en général réalisée intra-race sur les valeurs génétiques additives. Le progrès génétique réalisé intra-race n'aboutit pas forcément à une amélioration similaire des performances en croisement. En effet, lorsque le déterminisme génétique d'un caractère n'est pas purement additif, il n'y a en général pas identité entre les performances en race pure et en croisement. La réponse en croisement à une sélection en race pure est alors fonction de la corrélation génétique entre performances en race pure et en croisement

La sélection peut aussi théoriquement entraîner, du fait de modifications des fréquences géniques dans les races parentales, des changements de valeur de l'hétérosis. Cependant, les quelques résultats expérimentaux disponibles n'indiquent aucune modification 
des valeurs de l'hétérosis, même après plusieurs dizaines de générations de sélection.

\section{2 / Sélection de races spécialisées}

Comme nous l'avons vu, le croisement permet, par l'utilisation de types génétiques paternels et maternels spécialisés, de tirer profit des effets de complémentarité. Cette spécialisation se traduit par des contributions inégales à la productivité de l'étage terminal du croisement. Ainsi, dans le cas de la production de viande, la contribution de la race paternelle ne concerne que les effets directs sur la croissance, l'efficacité alimentaire, la quantité et la qualité de la viande. La contribution du type génétique maternel concerne les effets directs, mais aussi les effets maternels sur ces mêmes caractères et sa productivité numérique ou pondérale. Il en résulte généralement une spécialisation des objectifs de sélection . Dans le cas de la production de viande, les types génétiques paternels seront sélectionnés exclusivement pour l'efficacité de la croissance et la qualité du tissu maigre. Dans le cas des types génétiques maternels, la sélection portera également sur l'efficacité de la croissance et la qualité du tissu maigre, mais également sur les caractères de reproduction.

\section{3 / Sélection pour la performance en croisement}

L'hétérosis étant fonction de la distance génétique entre races, il peut être intéressant d'accroître par sélection la distance génétique entre deux populations pour augmenter l'hétérosis et la performance en croisement. C'est le principe de la sélection récurrente.Elle est théoriquement intéressante lorsque les caractères sélectionnés sont influencés par des effets de surdominance. Il en existe deux variantes. Dans le cas de la sélection récurrente réciproque, on améliore simultanément deux races $\mathrm{A}$ et $\mathrm{B}$ en sélectionnant les reproducteurs sur la base des performances de leurs descendants croisés AxB. Les reproducteurs sont ensuite accouplés en race pure pour produire la génération suivante. Dans le cas de la sélection récurrente sur population consanguine, une seule population est sélectionnée, l'autre étant une population consanguine en fonction de laquelle on veut améliorer la première. En pratique, la sélection récurrente est peu utilisée, sauf dans certaines firmes de sélection avicole.Une des raisons en est la nécessité de pratiquer une sélection familiale (en général sur descendance). Dans les espèces où la sélection est individuelle, elle en en général moins efficace du fait d'une diminution des intensités de sélection et d'un allongement de l'intervalle de génération. L'utilisation d'une sélection assistée par marqueurs pourrait changer cette situation. En effet, une évaluation précoce et individuelle des candidats devient alors théoriquement possible.

\section{Conclusion}

L'utilisation du croisement comme méthode d'amélioration génétique recouvre des aspects extrêmement variés. Cette diversité concerne à la fois les objectifs du croisement et les modalités de son utilisation, qui varient fortement en fonction de de la production et de l'espèce considérées. Le choix d'un plan de croisement dépendra notamment de la variabilité géné- tique entre races des caractères économiquement les plus importants et de la productivité numérique de l'espèce. Dans de nombreux cas, ce choix ne peut en outre pas être dissocié de celui d'un plan de sélection et aboutit au problème plus général du choix d'un plan d'amélioration génétique.

\section{Références bibliographiques}

Bell A.E., 1972. More on reciprocal recurrent selection. In: Proceeding of the 21st National Poultry Breeders Roundtable, Poultry Breeders of America, Kansas City, Missouri.

Dickerson G.E., 1969. Experimental approaches in utilising breed resources. Anim. Breed. Abstr., 37, 191-202.

Dickerson G.E., 1973. Inbreeding and heterosis in animals. In: Proceedings of the Animal Breeding and Genetics Symposium in honor of Dr JL Lush, American Society of Animal Science and Dairy Science Association, Champaign, Illinois, pp 54-77.

Eisen E.J., 1989. Genetic models to predict crossbred performance: a review. Rev. Brasil. Genet., 12, (suppl), 1326.

Eisen E.J., Hörstgen-Schark G., Saxton A.M., Bandy T.R. 1983. Genetic interpretation and analysis of diallel crosses with animals. Theor. Appl. Genet., 65, 17-23.

Elsen J.M., Sellier P., 1978. Etude conjointe de l'intérêt de la sélection sur la prolificité et de l'utilisation d'une lignée mâle spécialisée chez le porc. Ann. Génét. Sél. Anim.,10, 403-441.

Hill W.G., 1971. Theoretical aspects of crossbreeding. Ann. Génét. Sél. Anim., 3, 23-34.

Hill W.G., 1982. Dominance and epistasis as components of heterosis. Z. Tierzüchtg. Züchtgsbiol., 99, 161-168.

Koch R.M., Dickerson G.E., Cundiff L.V., Gregory K.E., 1985. Heterosis retained in advanced generations of crosses among Angus and Hereford cattle. J. Anim. Sci., 60, 11171132 .

Moav R., 1966. Specialised sire and dam lines. III-Choice of the most profitable parental combination when component traits are genetically non-additive. Anim. Prod., 8, 365-374.

Moav R., Hill W.G., 1966. Specialised sire and dam lines. IV-Selection within lines. Anim. Prod., 8, 375-390.

Sellier P., 1982. Selecting populations for use in crossbreeding. In : 2nd World Congress on Genetics Applied to Livestock Production, Vol. VI, 15-49, Editorial Garsi, Madrid.

Sheridan A.K., 1981. Crossbreeding and heterosis. Anim. Breed. Abstr., 49, 131-144.

Willham R.L., Pollak E., 1985. Theory of heterosis. J. Dairy Sci., 68, 2411-2417. 